\title{
On-chip excitation of single germanium vacancies in nanodiamonds embedded in plasmonic waveguides
}

Hamidreza Siampour (1)', Shailesh Kumar (1)', Valery A. Davydov², Liudmila F. Kulikova², Viatcheslav N. Agafonov ${ }^{3}$ and Sergey I. Bozhevolnyi (1)

\begin{abstract}
Monolithic integration of quantum emitters in nanoscale plasmonic circuitry requires low-loss plasmonic configurations capable of confining light well below the diffraction limit. We demonstrated on-chip remote excitation of nanodiamond-embedded single quantum emitters by plasmonic modes of dielectric ridges atop colloidal silver crystals. The nanodiamonds were produced to incorporate single germanium-vacancy (GeV) centres, providing bright, spectrally narrow and stable single-photon sources suitable for highly integrated circuits. Using electron-beam lithography with hydrogen silsesquioxane (HSQ) resist, dielectric-loaded surface plasmon polariton waveguides (DLSPPWs) were fabricated on single crystalline silver plates to contain those of deposited nanodiamonds that are found to feature appropriate single GeV centres. The low-loss plasmonic configuration enabled the 532-nm pump laser light to propagate on-chip in the DLSPPW and reach to an embedded nanodiamond where a single GeV centre was incorporated. The remote GeV emitter was thereby excited and coupled to spatially confined DLSPPW modes with an outstanding figure-of-merit of 180 due to a six-fold Purcell enhancement, 56\% coupling efficiency and $\sim 33 \mu \mathrm{m}$ transmission length, thereby opening new avenues for the implementation of nanoscale functional quantum devices.
\end{abstract}

\section{Introduction}

Waveguides based on surface plasmon polariton (SPP) modes with inherent subwavelength confinement are fundamentally better than dielectric-based (and therefore diffraction-limited) photonic waveguides with respect to the available Purcell enhancement of the spontaneous emission rate from embedded quantum emitters ${ }^{1,2}$ and the current development trend towards integration and miniaturization $^{3-12}$. Different metal-dielectric configurations have been developed that support propagating plasmonic modes confined beyond the diffraction limit, allowing for

Correspondence: Hamidreza Siampour (hasa@mci.sdu.dk)

${ }^{1}$ Centre for Nano Optics, University of Southern Denmark, Campusvej 55, Odense M DK-5230, Denmark

${ }^{2}$ L.F. Vereshchagin Institute for High Pressure Physics, Russian Academy of Sciences, Troitsk, Moscow 142190, Russia

Full list of author information is available at the end of the article. strong light-matter interaction down to the single-photon level ${ }^{1,4,13-15}$. Various types of SPP-based structures, such as metal nanowires $(\mathrm{NW})^{16-19}$, parallel NWs ${ }^{20}$, V-grooves $(\mathrm{VGs})^{21}$ and wedge waveguides ${ }^{22}$, have been demonstrated to guide single plasmons, quanta of propagating plasmonic modes, for potential quantum applications. However, the practical realization of SPP-based integrated quantum photonics has remained elusive due to several formidable challenges, including high propagation losses of SPP modes and the rather limited control over interfacing with single quantum emitters. Recently, by using relatively low-loss dielectric-loaded SPP waveguides (DLSPPWs) structured on a silver (Ag) film, simple quantum plasmonic circuits composed of embedded nanodiamonds with nitrogen-vacancy (NV) centres have been demonstrated $^{23,24}$. The nanodiamonds hosting colour centres with their photostable single photon emission and 


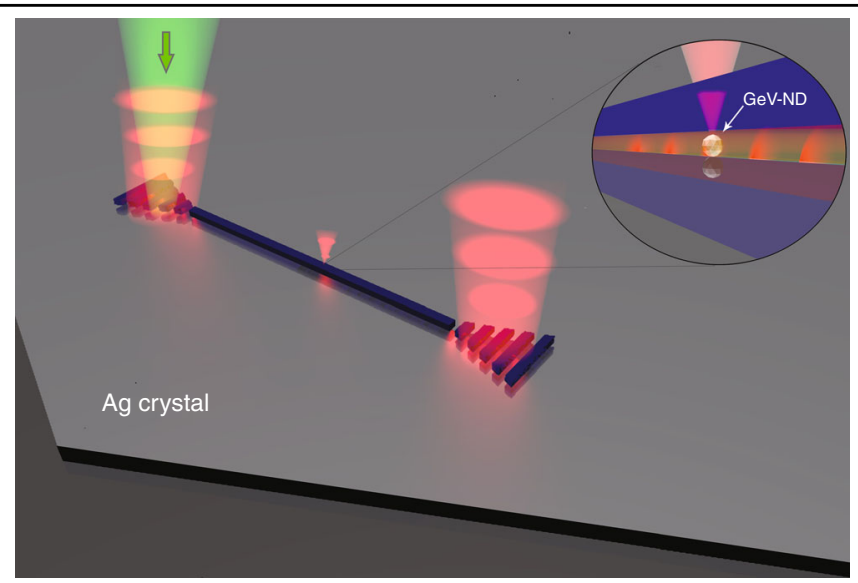

Fig. 1 Schematic of the device layout and working principle for on-chip excitation of a nanodiamond (ND) carrying spectrally narrow single GeV quantum emitters embedded in a DLSPP waveguide. A 532-nm pump laser light is coupled with a grating, propagates on-chip in the low loss DLSPPW and reaches an embedded nanodiamond that contains a single GeV centre (GeV-ND). The remote GeV emitter is thereby excited, generating single optical plasmons propagating along the waveguide and outcoupling from the ends

optically readable spin states are promising candidates to build integrated quantum devices, for example, integration into plasmonic circuits. In addition to NV centres, a family of diamond colour centres based on group-IV elements in the periodic table, i.e. silicon-vacancy $(\mathrm{SiV})^{25-31}$, germanium-vacancy $(\mathrm{GeV})^{32-36}$ and tin-vacancy $(\mathrm{SnV})$ centres $^{37,38}$, have attracted attention due to their structural symmetries, leading to high emission into zero-phonon lines (ZPLs) accompanied by bright, spectrally narrow emission lines and indistinguishability of photons emitted from different emitters. The SiV centres exhibit an optical transition at a longer wavelength (ZPL at $738 \mathrm{~nm}$ ) operating with smaller SPP loss in the metal ${ }^{39}$. At the same time, the SiV excited state decay is dominated by the nonradiative relaxation, causing lower quantum efficiency for $\mathrm{SiV}$ centres ${ }^{40}$. More recently, the $\mathrm{GeV}$ centres in diamond have shown higher quantum efficiency and a larger absorption cross section than $\mathrm{SiV}$ centres $^{33}$. Having such single-photon emitters in diamond nanocrystals becomes very important when it comes to the implementation of hybrid quantum-plasmonic systems and can facilitate remote excitation of $\mathrm{GeV}$ centres incorporated in a plasmonic chip.

In this work, we demonstrate on-chip generation and propagation of spectrally narrow single optical plasmons excited by $\mathrm{GeV}$ centres in nanodiamonds using DLSPPWs. The synthesis and detailed characterization of the $\mathrm{GeV}$ nanodiamonds are presented here, for the first time to our knowledge, describing specific features useful for working with these colour centres. The nanodiamonds were produced using the high-pressure high-temperature (HPHT) method, and Ge was introduced during the growth process to incorporate single $\mathrm{GeV}$ centres (see details in the Materials and methods section). Furthermore, we propose and demonstrate a hybrid approach using DLSPPWs structured on single Ag crystals that feature considerably lower SPP damping rates in comparison with Ag films fabricated by other techniques ${ }^{41-47}$. The latter allows us to realize sufficiently long SPP propagation at both the excitation and emission wavelengths of $\mathrm{GeV}$ centres, thereby facilitating the remote excitation of $\mathrm{GeV}$ centres in nanodiamonds incorporated in a plasmonic chip. The colloidal Ag crystal flakes were synthesized using a recently reported method ${ }^{47}$. Figure 1 shows a schematic of the device layout, introducing the working principle of on-chip remote excitation. The hybrid plasmonic configuration enabled the green laser light to propagate on-chip, in the DLSPPW, and reach to an embedded nanodiamond containing a single $\mathrm{GeV}$ centre. The remote $\mathrm{GeV}$ emitter is thereby excited, and single photons are channelled to a DLSPPW mode. Finally, the ability of GeV-DLSPPW system for efficient long-range delivery is compared with other hybrid quantum plasmonic systems, revealing an exceptional figure of merit (FOM) of 180 due to a $\sim$ six-fold Purcell enhancement, $\sim 56 \%$ coupling efficiency and $\sim 33 \mu \mathrm{m}$ transmission length at $\lambda=602 \mathrm{~nm}$ (ZPL, GeV).

\section{Results}

A scanning electron microscopy (SEM) image of the $\mathrm{GeV}$ nano and microdiamonds in the 'raw' sample after HPHT synthesis is shown in Fig. 2a. The inset shows a transmission electron microscopy (TEM) image of the $\mathrm{GeV}$ nanodiamonds of different sizes (from 20 to $120 \mathrm{~nm}$ ) taken after the chemical and ultrasonic treatment. Chemical treatment was carried out with three highly concentrated acids, $\mathrm{HNO}_{3}, \mathrm{HClO}_{4}$ and $\mathrm{H}_{2} \mathrm{SO}_{4}$ (at $200{ }^{\circ} \mathrm{C}$ for $3 \mathrm{~h}$ ), to remove traces of graphite. The ultrasonic treatment was done with a UP200H device (Hielscher). The Ge atom is located in the middle of two empty lattice sites, 


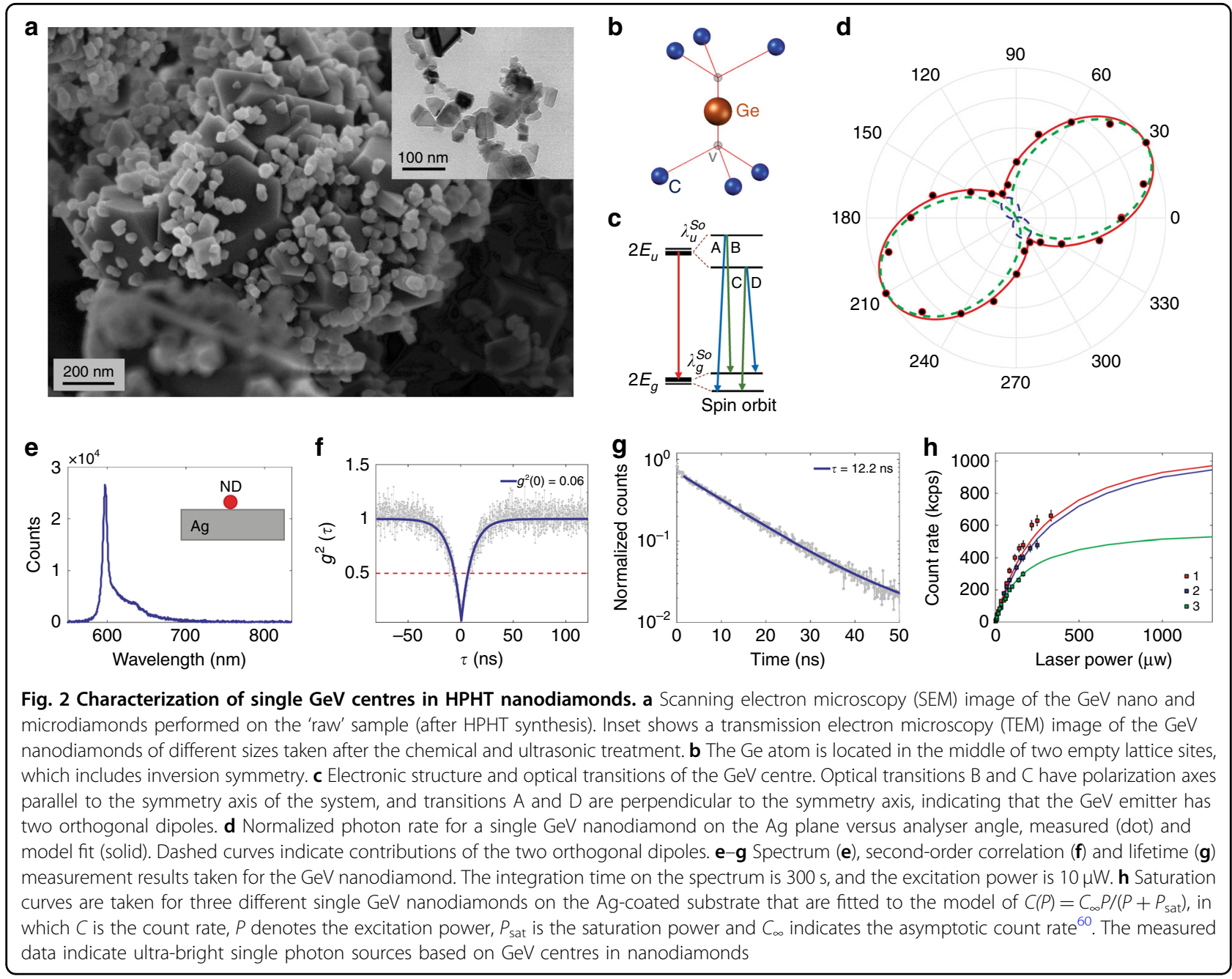

which includes inversion symmetry (Fig. 2b), and the system has an electronic structure and optical transitions (Fig. 2c) similar to reports for the group-IV family of diamond colour centres ${ }^{29,33,37,48}$.

In the first designed experiment, a Ag-coated silicon wafer was patterned with regularly placed gold mar$\operatorname{kers}^{23,24}$, and the synthesized $\mathrm{GeV}$ nanodiamonds were spin coated onto this substrate. A 1-nm layer of poly (allylamine hydrochloride) (PAH) was put on the $\mathrm{Ag}$ layer to improve the distribution and attachment of the nanodiamonds to the Ag surface ${ }^{49}$. The sample was then raster scanned using confocal fluorescence microscopy. Lifetime (Fig. 2g), spectrum (Fig. 2e), autocorrelation (Fig. 2f) and saturation curve (Fig. 2h) measurements were taken for the single $\mathrm{GeV}$ nanodiamonds on the $\mathrm{Ag}$ film. The results indicate ultrabright, spectrally narrow and stable single photon sources in the nanodiamonds. We also measured the polarization characteristics of $\mathrm{GeV}$ nanodiamonds deposited on a Ag-coated substrate, using an analyser in the detection pathway to determine the projection of polarization axes of single photons emitted on the surface plane (see the experimental setup in Supplementary Materials, Figure S1). The measured polarization data for a single $\mathrm{GeV}$ nanodiamond are shown in Fig. 2d and well-fitted to the model of two orthogonal dipoles, following the polarization characteristics of the group-IV colour centres ${ }^{28,50}$.

The position of nanodiamonds containing single $\mathrm{GeV}$ centres was determined with respect to the gold markers. Using electron beam lithography with hydrogen silsesquioxane (HSQ) resist ${ }^{23}$, waveguides are fabricated on Ag-coated substrate to contain those nanodiamonds that are found to feature appropriate single $\mathrm{GeV}$ centres. Controlled placement of nanodiamonds in plasmonic nanostructures is explained in the Supplementary Materials (Figure S6). Our deterministic technology provides a $\sim 30 \mathrm{~nm}$ precision in placement that can further be enhanced by using the SEM image. This is limited by the size of nanodiamonds, which can be reduced down to $1 \mathrm{~nm}$, achieved by current diamond synthesis technology ${ }^{51,52}$. A schematic of the device layout for on-chip direct excitation of nanodiamonds is 
a
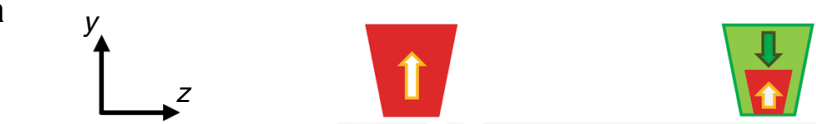

1

1 म म

पामा

$\begin{aligned} \text { ND } & 0 \\ \text { HSQ } & \\ \text { Ag film } & \end{aligned}$

$\mathrm{Si}$

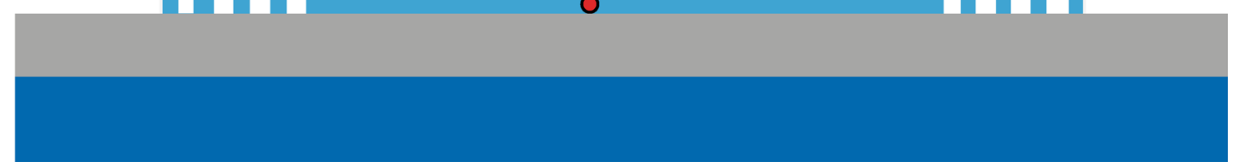

b
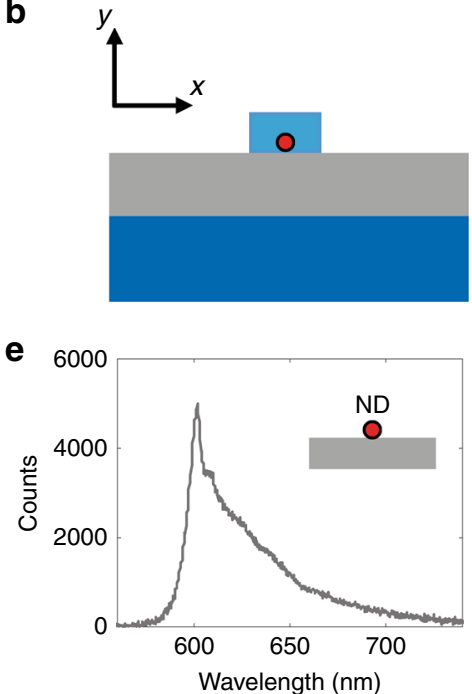

h

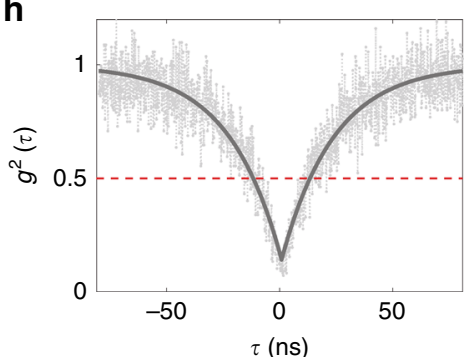

C

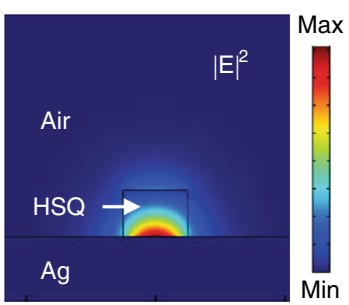

f

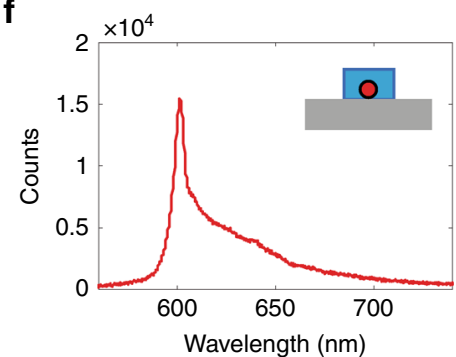

i

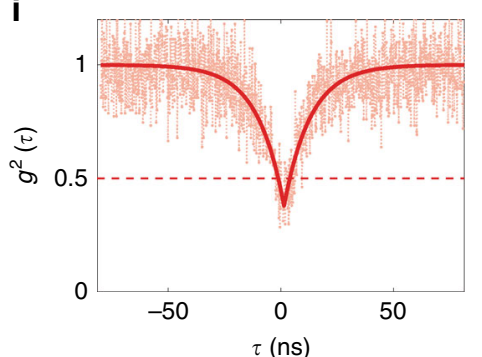

d
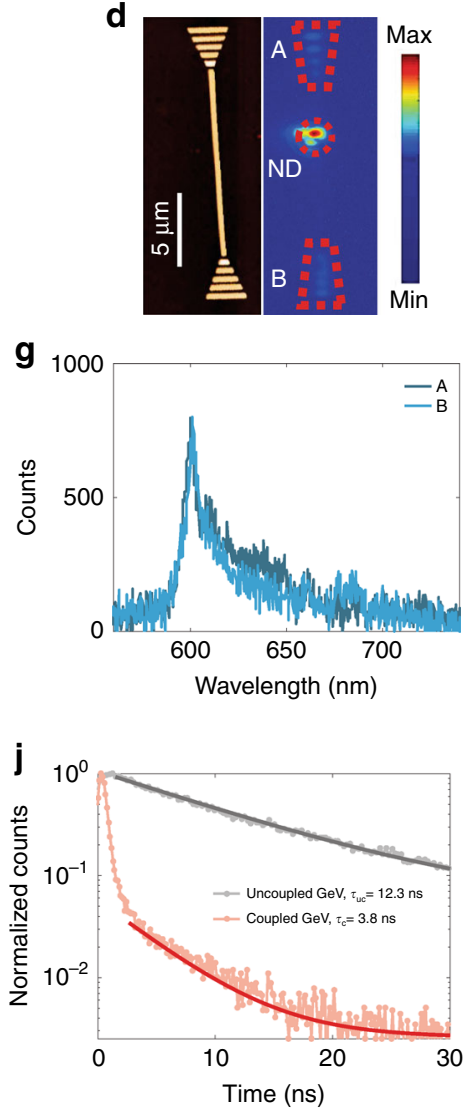

Fig. 3 On-chip direct excitation of a single GeV nanodiamond. a, b Schematic of a sample layout and the working principle of direct excitation of a GeV nanodiamond embedded in a plasmonic waveguide. c Simulated mode profile for the DLSPP waveguide at $\lambda=602 \mathrm{~nm}$ (GeV ZPL). d AFM image of the fabricated waveguide (left), and CCD camera image of the whole structure where the nanodiamond is excited and a fluorescence image of the focal plane is taken (right). $\mathbf{e}-\mathbf{g}$ Spectrum taken from uncoupled GeV (e), coupled GeV (f) and outcoupled light through the grating ends A and B (g). The integration time of the spectra is $300 \mathrm{~s}$, and the excitation power is $25 \mu \mathrm{W}$. $\mathbf{h}, \mathbf{i}$ Second order correlation of the $\mathrm{GeV}$ centre before (h) and after (i) coupling to the waveguide. $\mathbf{j}$ Lifetime of the GeV centre taken before (grey) and after (red) coupling

illustrated in Fig. 3a, and Fig. 3b, c shows a distribution of normalized $|E|^{2}$ (where $E$ is the electric field) of the DLSPPW mode with a cross-section of $180 \mathrm{~nm}$ in height and $250 \mathrm{~nm}$ in width of HSQ (dielectric constant of 1.41) on the Ag surface ${ }^{53}$ at $\lambda=602 \mathrm{~nm}$. An atomic force microscopy (AFM) image of the HSQ waveguide is presented in Fig. 3d (left). The whole structure, when the $\mathrm{GeV}$ centre was directly excited with green pump laser, was imaged using a charge coupled device (CCD) camera. The image shows three spots, ND, A and B (Fig. 3d, right). This shows excitation and emission of the $\mathrm{GeV}$ emitter
(ND), coupling of GeV emission to the DLSPPW mode, propagation and out-coupled radiation from the two ends (A and B). The emission spectra of the uncoupled (Fig. 3e) and coupled GeV (Fig. 3f) indicate a $\sim 3$ times enhancement in the emission rate of the $\mathrm{GeV}$ centre after coupling to the confined DLSPPW mode (for the same excitation power of $25 \mu \mathrm{W}$ and an integration time of $300 \mathrm{~s}$ ). Figure $3 g$ shows the spectra from the out-coupling grating couplers $A$ and $B$, indicating the on-chip transmission of the spectrally narrow surface plasmon mode excited by the $\mathrm{GeV}$ emitter. The antibunching dip of the correlation 

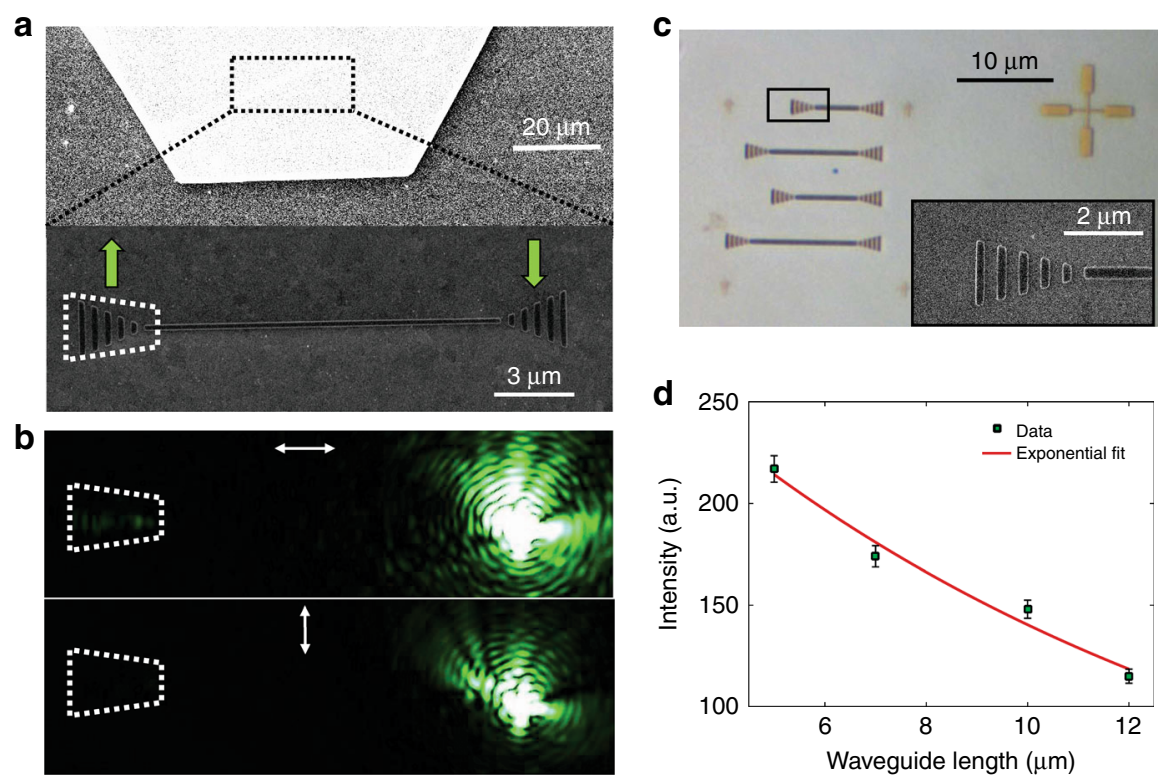

Fig. 4 Transmission of the 532-nm pump laser light along the low-loss plasmonic waveguide. a SEM image of a single crystalline Ag flake (top) and fabricated DLSPP waveguide on the Ag plate (bottom). $\mathbf{b}$ Optical characterization of the waveguide for parallel (top) and perpendicular (bottom) polarizations of 532-nm laser light. c Bright-field microscopy image of fabricated waveguides on the Ag flake with different lengths. The inset shows an SEM image of the grating couplers at the end of the waveguides with an optimized taper opening angle of $30^{\circ}$ that maximize the in-coupling efficiency of DLSPPWs (see details in refs. ${ }^{54,55}$ ). $\mathbf{d}$ Measured data for propagation characteristics of the DLSPPWs on Ag crystals with different lengths (green squares) and exponential fitting curve (red line), giving the propagation length of $11.8 \mu \mathrm{m}$ at $\lambda=532 \mathrm{~nm}$ (pump laser) for the DLSPPW on the Ag flake

function, before (Fig. 3h) and after (Fig. 3i) coupling, indicates a single $\mathrm{GeV}$ emitter $\left(g^{2}(\tau=0)<0.5\right)$. The data are analysed with a single exponential fitting curve ${ }^{33}$. The lifetime of the $\mathrm{GeV}$ centre before (Fig. 3j, grey) and after (Fig. 3j, red) coupling is measured, indicating a reduction (from $\sim 12.3$ to $\sim 3.8 \mathrm{~ns}$ ) for the coupled $\mathrm{GeV}$ centre. The lifetimes are obtained by tail fitting of the measured data with a single exponential curve ${ }^{23}$. The lifetime decreased by a factor of $\sim 3 \pm 0.5$, which is in addition to the two times reduction due to the Ag plane (Supplementary Materials, Figure S2), giving, on average, a $\sim$ sixfold Purcell enhancement.

We estimated the propagation length of the DLSPPW on the Ag film using the fluorescence intensities at the two ends of the waveguide ${ }^{23}$. We obtain the propagation length of $9 \pm 3 \mu \mathrm{m}$ for the GeV-DLSPPW hybrid system on the $\mathrm{Ag}$ film, which is smaller than the NV-DLSPPW system ${ }^{23}$ due to the higher SPP loss at the lower wavelength region corresponding to emission from $\mathrm{GeV}$ centres.

Using a single-crystalline Ag flake instead of the Ag film significantly enhanced the DLSPPW propagation length (Fig. 4). Figure 4a shows an SEM image of a HSQ waveguide fabricated on a Ag crystal flake. Optical characterization of the waveguide shows transmission of green laser light $(532 \mathrm{~nm}$ ) through the DLSPPW mode for the polarization along the waveguide axis (Fig. 4b). We have measured the transmission for several waveguides with different lengths (Fig. 4c, d), achieving an extraordinary long propagation length of $\sim 11.8 \mu \mathrm{m}$ for the green laser light through the low-loss DLSPPW (see Supplementary Figure S4 for the characterization of DLSPPW without grating couplers). The funnel tapering dimensions of the grating coupler (Fig. 4c, inset) are optimized to obtain the in-coupling efficiency of $\sim 12 \%$ extracted from the fitting curve shown in Fig. 4d using the well-established cutback method ${ }^{54,55}$.

In the second designed experiment, we employ the capability of green light transmission in the DLSPPW on $\mathrm{Ag}$ crystals and demonstrate remote excitation of $\mathrm{GeV}$ nanodiamonds embedded in low-loss DLSPPWs. We spin-coated a small amount of the synthesized solution of Ag crystal flakes on a Ag-coated silicon wafer. A PAH layer is put on the Ag film to improve the adhesion of the Ag flakes to the substrate ${ }^{49}$. The markers are fabricated on $\mathrm{Ag}$ flakes, and the nanodiamonds with $\mathrm{GeV}$ centres are spin-coated. The sample fluorescence is then imaged using confocal microscopy. Spectra and correlation functions are taken for the nanodiamonds on $\mathrm{Ag}$ crystals. Using electron beam lithography, the HSQ waveguides are fabricated on the Ag crystal, embedding the selected single $\mathrm{GeV}$ nanodiamonds. A schematic of the device layout and working principle for on-chip remote excitation is illustrated in Fig. 5a. Figure 5b shows an AFM image of the fabricated waveguide (left) and a 

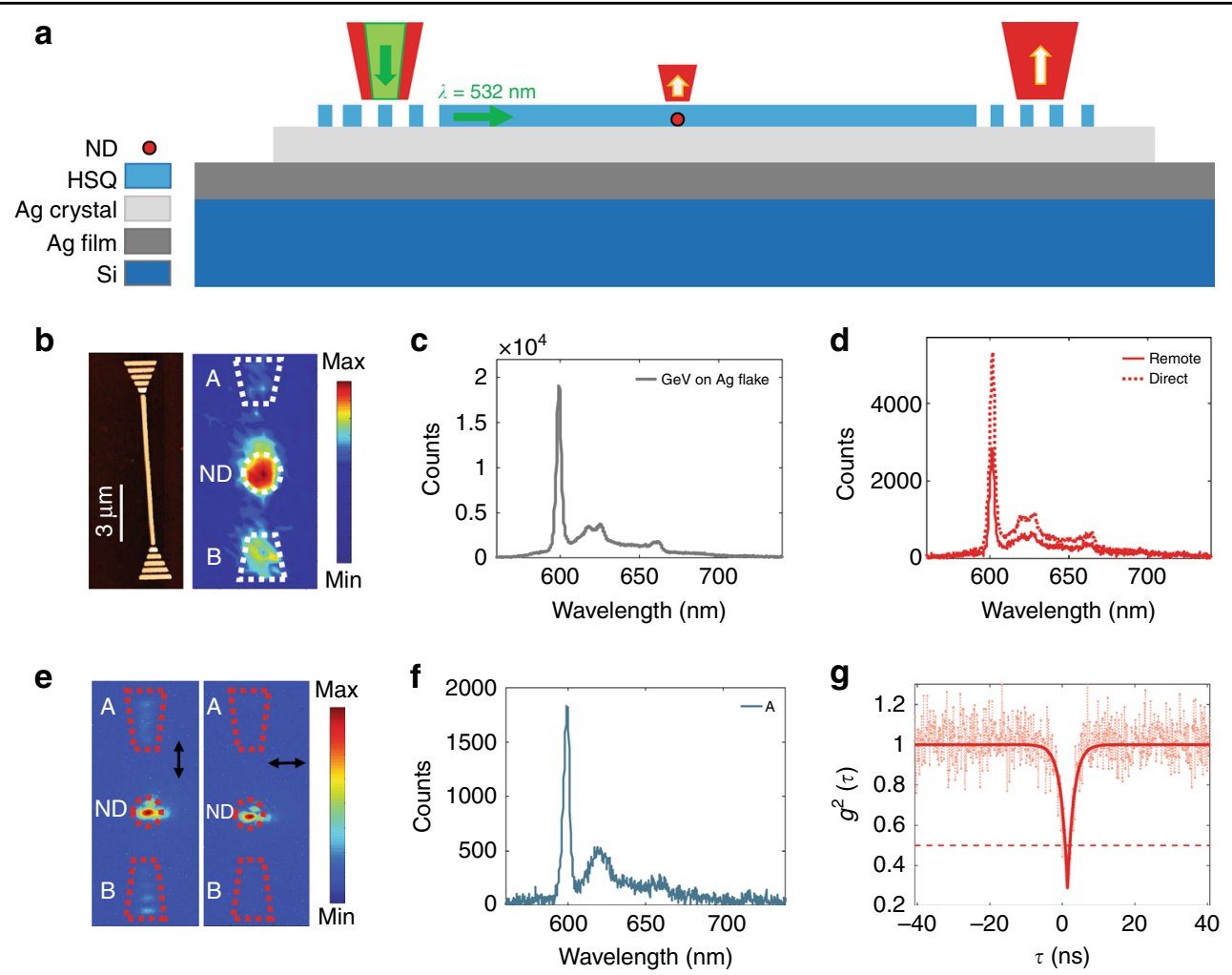

Fig. 5 On-chip remote excitation of a single GeV nanodiamond. a Schematic of a sample layout for on-chip remote excitation of a GeV nanodiamond embedded in the plasmonic structure. $\mathbf{b}$ AFM image of the fabricated waveguide (b, left) and galvanometric mirror scan image showing the remote excitation of the embedded GeV where the pump laser light is illuminated at end B (b, right). Higher emission at end B is caused by the background fluorescence from the grating coupler exposed to the strong pump light. c, $\mathbf{d}$ Spectra taken from the uncoupled GeV, i.e. the nanodiamond on the Ag plate (c) and from coupled GeV when excited remotely (d, solid line) and in the case of direct excitation (d dotted line). e CCD images for the coupled system when excited directly and with a linear polarizer placed in the detection path are presented for two orthogonal polarizations, parallel (left) and perpendicular (right) to the waveguide axis. $\mathbf{f}$ Spectrum taken from the outcoupled light through grating end $\mathrm{A}$ in the case of remote excitation. The integration time on the spectra data is $300 \mathrm{~s}$, and the excitation powers are $2 \mu \mathrm{W}(\mathbf{c}, \mathbf{d})$ and $5 \mu \mathrm{W}(\mathbf{f})$. g Second order correlation function of the GeV emitter confirming a single photon emission

galvanometric mirror scan image (right) when the waveguide is excited from end $B$ with the green pump laser and the fluorescence emission detected at the focal plane. Emission from the embedded $\mathrm{GeV}$ nanodiamond located inside the waveguide confirms a remote excitation of the $\mathrm{GeV}$ centre, and out-coupled radiation from the two ends (A and $\mathrm{B}$ ) indicates the coupling of the $\mathrm{GeV}$ centre to the DLSPPW mode. Figure $5 \mathrm{c}$ illustrates the spectrum of the $\mathrm{GeV}$ emitter before coupling (nanodiamond on the Ag crystal). The emission spectra after coupling for the remotely excited $\mathrm{GeV}$ centre (solid line) and the spectrum for the same $\mathrm{GeV}$ centre when excited directly (dotted line) is presented in Fig. 5d. CCD images for the coupled system when excited directly and with a linear polarizer placed in the detection path are presented in Fig. 5e. A comparison of the two images in Fig. 5e clearly indicates a strongly polarized emission from the end of the waveguide due to the coupling of the emission to the fundamental transverse magnetic (TM) mode of the DLSPP waveguide, propagation and subsequent scattering from the ends. Figure $5 f$ shows the spectrum measured at the grating end $\mathrm{A}$, when the $\mathrm{GeV}$ centre is remotely excited (Fig. 4b). In Fig. 5g, we present the second-order correlation function for the $\mathrm{GeV}$ centre, confirming single photon emission $\left(g^{2}(\tau=0)<0.5\right)$. Apparent differences in the second-order correlation functions shown in Figs. $3 \mathrm{i}$ and $5 \mathrm{~g}$ are related to a large dispersion in the fluorescence lifetimes of the $\mathrm{GeV}$ centres in the nanodiamonds (Supplementary Figure 2a).

We simulated the GeV decay rate into the DLSPPW mode using the finite-element modelling (FEM) method $^{23,56}$. The $\mathrm{GeV}$ centre was modelled as a single dipole emitting at $602 \mathrm{~nm}$ in the simulations. The effect of the orientation of the $\mathrm{GeV}$ dipole axis on the decay channels is discussed in the Supplementary Materials (Figure S3). An up to four-fold decay rate to the plasmonic mode is predicted for a $\mathrm{GeV}$ centre in the waveguide compared to its emission in vacuum. Figure 6a shows the plasmonic decay rate dependence for the optimum orientation of the dipole on its position in the 

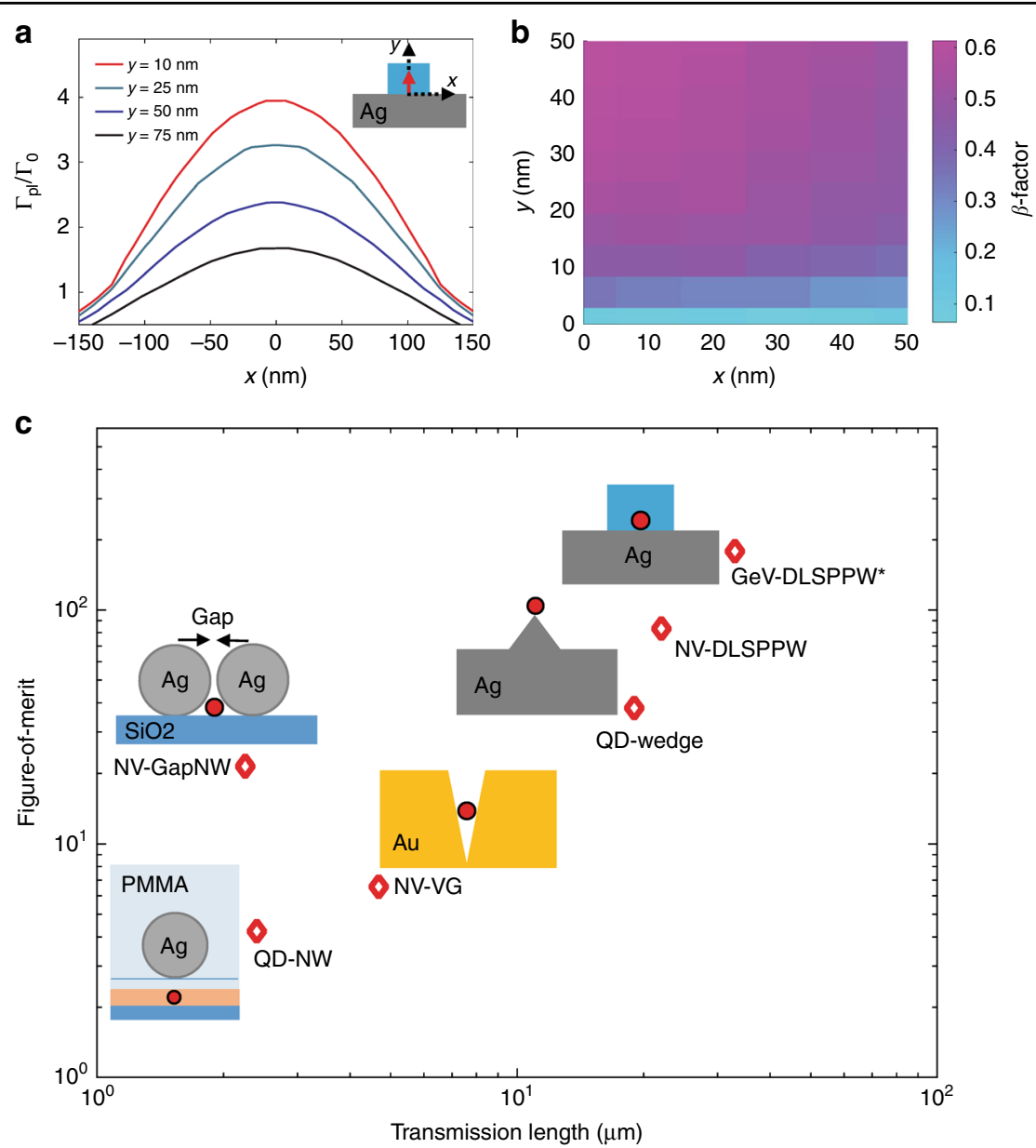

Fig. 6 Efficiency of the GeV-DLSPPW platform compared with other hybrid quantum systems. a Simulated plasmonic decay rate $\left(\Gamma_{\mathrm{p}} / \Gamma_{0}\right)$ for the DLSPPW coupled GeV centre. Inset shows the cross section of a $y$-oriented dipole emitter inside the DLSPP waveguide (top right). $\mathbf{b}$ Distribution profile of the $\beta$-factor, i.e. $\Gamma_{\mathrm{p}} / \Gamma_{\text {tot }}$ for a distribution of the $\mathrm{GeV}$ centre inside a nanodiamond, where each coloured square represents the central value of the corresponding in-plane dipole position. c Figure-of-merit (FOM) and transmission length of hybrid quantum plasmonic systems. The FOM of GeV-DLSPPW on the Ag crystal is compared with other demonstrated quantum emitter-plasmonic waveguide (QE-PW) hybrid systems, including quantum dot-Ag nanowire (QD-NW) ${ }^{16}, \mathrm{NV}$-Gap Ag nanowire (NV-GapNW) ${ }^{20}, \mathrm{NV}-\mathrm{V}$ groove channel waveguides (NV-VG) ${ }^{21}$, QD-Wedge waveguides (QD-wedge) ${ }^{22}$ and NV-DLSPPW on a Ag film ${ }^{23}$. The black diamond markers in the graph are extracted from the experimental results reported for the corresponding hybrid systems

waveguide. The emission efficiency ( $\beta$ factor) of the emitter to the DLSPPW mode is given by $\beta=\Gamma_{\mathrm{pl}} / \Gamma_{\text {tot }}$, where $\Gamma_{\text {tot }}$ denotes the total decay rate, and $\Gamma_{\mathrm{pl}}$ is plasmonic decay rate ${ }^{23}$. The total decay rate is calculated from the time evolution of the total dissipated power ${ }^{23,56,57}$. The $\beta$ factor for a $y$-oriented $\mathrm{GeV}$ coupled to a DLSPPW is simulated as a function of the position in the crosssection (Fig. 6b). Palik's data ${ }^{53}$ are used for modelling of the Ag refractive index. The simulated results show that the $\beta$ factor can reach $62 \%$. We measure the apparent $\beta$ factor $\left(\beta_{\mathrm{pl}}\right)$ using $\beta_{\mathrm{pl}} \simeq\left(I_{\mathrm{A}}+I_{\mathrm{B}}\right) /\left(I_{\mathrm{A}}+I_{\mathrm{B}}+I_{\mathrm{GeV}}\right)$, in which $I_{\mathrm{A}}$ and $I_{\mathrm{B}}$ are the out-coupled radiation at the ends $\mathrm{A}$ and $\mathrm{B}$, respectively, and $I_{\mathrm{GeV}}$ is the measured intensity at the $\mathrm{GeV}$ centre position ${ }^{23}$. This gives a $\beta$ factor of $56 \%$ for the GeV-DLSPPW system shown in Fig. 5, which is in good agreement with the simulated $\beta$ factor, indicating accurate alignment of the waveguide with respect to the $\mathrm{GeV}$ nanodiamond. We measured the $1 / e$ propagation length of the $\mathrm{GeV}$ emission along the DLSPPW on Ag crystal, the same way as described for $\mathrm{Ag}$ film, and obtained a value of $33 \pm 3 \mu \mathrm{m}$ (Supplementary S5). This is significantly larger than DLSPPW on the polycrystalline Ag film and even higher than the propagation length of NV-DLSPPW ${ }^{23}$, indicating low material loss for the single crystalline Ag flakes.

\section{Discussion}

The ability of a hybrid plasmonic system to realize efficient single-photon transmission can be characterized with the figure of merit $\left(\mathrm{FOM}=1 / \lambda \beta L_{\mathrm{P}} \Gamma_{\mathrm{tot}} / \Gamma_{0}\right)^{21,23}$. The $\mathrm{GeV}$-DLSPPW hybrid system reaches a FOM value of $180 \pm 25\left(\Gamma_{\text {tot }} / \Gamma_{0}=6 \pm 1, \beta=0.56 \pm 0.03, L_{\mathrm{P}}=33 \pm 3 \mu \mathrm{m}\right.$ 
and $\lambda=602 \mathrm{~nm}$ ), clearly outperforming previous demonstrations of quantum emitter-plasmonic waveguide (QE-PW) coupled systems ${ }^{16,20-23}$. A careful comparison of GeV-DLSPPW on the Ag crystal with other hybrid systems of QE-PW is presented in Fig. 6c. The efficiency of the light-matter interaction in the GeV-DLSPPW platform can also be compared to other colour centre photonic platforms using the so-called cooperativity parameter (defined as $\Gamma_{w} /\left(\Gamma_{\text {tot }}-\Gamma_{w}\right)$, in which $\Gamma_{w}$ is the decay rate into a waveguide mode). For the SiV-centre incorporated cavity system described in ref. ${ }^{25}$ and for the $\mathrm{GeV}$-based platform presented in ref. ${ }^{33}$, the cooperativity of $C=1$ and $C=0.1$ have been deduced, respectively. The cooperativity was estimated to be $C=1.5$ in this work using the GeV-DLSPPW platform, which should be understood as the upper cooperativity limit evaluated from our experiment at room temperature (as opposed to the cooperativity estimated at low temperatures ${ }^{25,33}$ ). This can be enhanced further by using the waveguideintegrated cavity resonator ${ }^{24}$ and/or using dielectric ridges with a larger refractive index (e.g. $\mathrm{TiO}_{2}$ with a refractive index of $\sim 2.4$ ) and smaller cross section DLSPPW mode (and therefore stronger coupling).

In conclusion, we have demonstrated on-chip generation and transmission of spectrally narrow single optical plasmons excited by $\mathrm{GeV}$ nanodiamonds embedded in DLSPPWs. The extraordinary long propagation length for the green pump laser has been achieved with DLSPPWs on Ag crystals, enabling thereby the remote excitation of $\mathrm{GeV}$ centres through propagating DLSPPW mode in a plasmonic chip. The performance of the GeV-DLSPPW hybrid system with respect to efficient single-photon transmission has been quantified with the FOM of 180 by a six-fold Purcell enhancement, 56\% coupling efficiency and $\sim 33 \mu \mathrm{m}$ transmission length, which indicates a superior performance in comparison with the previously demonstrated systems. Further enhancement of the Purcell factor is possible by using a larger refractive index dielectric (for example, $\mathrm{TiO}_{2}$ with a refractive index of $\sim 2.4$ at the wavelength of $600 \mathrm{~nm}$ ) with smaller dimensions in the DLSPPW cross section. Our demonstration opens the way for the integration of an excitation laser, quantum emitter and plasmonic circuit on the same chip. Detection of single plasmons and two-plasmon interference have already been demonstrated on a chip ${ }^{58,59}$. With the combination of all these technologies, it will be possible, in the near future, to have all the elements of a quantum plasmonic circuit integrated on a chip.

\section{Materials and methods}

\section{$\mathrm{GeV}$ nanodiamond synthesis}

HPHT synthesis of nanodiamonds with $\mathrm{GeV}$ centres has been realized based on the hydrocarbon metal catalystfree growth system. Tetraphenylgermanium $\mathrm{C}_{24} \mathrm{H}_{20} \mathrm{Ge}$
(Sigma-Aldrich) was used as the initial germaniumcontaining hydrocarbon compound. The synthesis was performed in a high-pressure apparatus of the Toroid type. Cylindrical samples of the initial material $(5 \mathrm{~mm}$ in diameter and $3 \mathrm{~mm}$ in height) obtained by cold pressing were put into graphite containers serving as heaters for the high-pressure apparatus. The containers were loaded in the apparatus up to $8 \mathrm{GPa}$ and heated up to the synthesis temperature under a constant load for 1-5 s. The obtained diamond products were then isolated and characterized with X-ray diffraction, Raman spectroscopy, SEM and TEM. The results of such characterization of the obtained products, which are mixtures of ultranano-, nano- and submicrometre-size fractions of diamond, show high, practically $100 \%$, yield in the formation of diamond. Size-fractional separation of diamonds was carried out in several stages that consisted of ultrasonically dispersing the diamond particles using UP200Ht dispersant (Hielscher Ultrasonic Technology), chemical treatment of the samples in a mixture of three acids $\left(\mathrm{HNO}_{3}-\mathrm{HClO}_{4}-\mathrm{H}_{2} \mathrm{SO}_{4}\right)$, and subsequent centrifugation of an aqueous or alcohol dispersion of diamond powders.

\section{Acknowledgements}

This work was supported by the European Research Council (ERC), Advanced Grant 341054 (PLAQNAP). V.A.D. and L.F.K. thank the Russian Foundation for Basic Research (Grant No. 18-03-00936) for financial support. H.S. thanks

Dr. Yuanqing Yang for his assistance in simulations.

\section{Authors' contributions}

H.S., S.K. and S.I.B. conceived the experiment. H.S. performed the measurements, structure fabrication and numerical computations. S.K. synthesized the colloidal silver crystals and assisted with the optical characterization. V.A.D., L.F.K. and V.N.A. supplied the GeV nanodiamond samples and performed scanning (SEM) and transmission (TEM) electron microscopy for the nanodiamond samples. H.S., S.K. and S.I.B. discussed the results obtained. H.S. wrote the manuscript with contributions from all authors. All authors reviewed the manuscript.

\section{Author details}

${ }^{1}$ Centre for Nano Optics, University of Southern Denmark, Campusvej 55, Odense M DK-5230, Denmark. ${ }^{2}$ L.F. Vereshchagin Institute for High Pressure Physics, Russian Academy of Sciences, Troitsk, Moscow 142190, Russia. ${ }^{3}$ GREMAN, Université de Tours, UMR CNRS CEA 6157, 37200 Tours, France

Conflict of interest

The authors declare that they have no conflict of interest.

Supplementary information is available for this paper at https://doi.org/ 10.1038/s41377-018-0062-5.

Received: 31 January 2018 Revised: 22 July 2018 Accepted: 15 August 2018 Accepted article preview online: 24 August 2018

Published online: 12 September 2018

\section{References}

1. Bozhevolnyi, S. I. \& Khurgin, J. B. The case for quantum plasmonics. Nat. Photonics 11, 398-400 (2017).

2. Bozhevolnyi, S. I. \& Khurgin, J. B. Fundamental limitations in spontaneous emission rate of single-photon sources. Optica 3, 1418-1421 (2016). 
3. Ozbay, E. Plasmonics: merging photonics and electronics at nanoscale dimensions. Science 311, 189-193 (2006).

4. Zia, R., Schuller, J. A., Chandran, A. \& Brongersma, M. L. Plasmonics: the next chip-scale technology. Mater. Today 9, 20-27 (2006).

5. Bozhevolnyi, S. I., Volkov, V. S., Devaux, E., Laluet, J. Y. \& Ebbesen, T. W. Channel plasmon subwavelength waveguide components including interferometers and ring resonators. Nature 440, 508-511 (2006).

6. Bermúdez-Ureña, E. et al. Plasmonic waveguide-integrated nanowire laser. Nano Lett. 17, 747-754 (2017).

7. Liu, Y., Zhang, J. S., Liu, H. P., Wang, S. \& Peng, L. M. Electrically driven monolithic subwavelength plasmonic interconnect circuits. Sci. Adv. 3 e1701456 (2017).

8. Bozhevolnyi, S. I. \& Mortensen, N. A. Plasmonics for emerging quantum technologies. Nanophotonics 6, 1185-1188 (2017).

9. Patel, R. N. et al. Efficient photon coupling from a diamond nitrogen vacancy center by integration with silica fiber. Light Sci. Appl. 5, e16032 (2016).

10. Kewes, $\mathrm{G}$. et al. A realistic fabrication and design concept for quantum gates based on single emitters integrated in plasmonic-dielectric waveguide structures. Sci. Rep. 6, 28877 (2016).

11. Lombardi, P. et al. Photostable molecules on chip: integrated sources of nonclassical light. ACS Photonics 5, 126-132 (2018)

12. Türschmann, $P$. et al. Chip-based all-optical control of single molecules coherently coupled to a nanoguide. Nano Lett. 17, 4941-4945 (2017).

13. Gramotnev, D. K. \& Bozhevolnyi, S. I. Plasmonics beyond the diffraction limit. Nat. Photonics 4, 83-91 (2010).

14. Tame, M. S. et al. Quantum plasmonics. Nat. Phys. 9, 329-340 (2013).

15. Fang, Y. R. \& Sun, M. T. Nanoplasmonic waveguides: towards applications in integrated nanophotonic circuits. Light Sci. Appl. 4, e294 (2015).

16. Akimov, A. V. et al. Generation of single optical plasmons in metallic nanowires coupled to quantum dots. Nature 450, 402-406 (2007).

17. Kolesov, R. et al. Wave-particle duality of single surface plasmon polaritons. Nat. Phys. 5, 470-474 (2009).

18. Kumar, S., Kristiansen, N. I., Huck, A. \& Andersen, U. L. Generation and controlled routing of single plasmons on a chip. Nano Lett. 14, 663-669 (2014).

19. Huck, A., Kumar, S., Shakoor, A. \& Andersen, U. L. Controlled coupling of a single nitrogen-vacancy center to a silver nanowire. Phys. Rev. Lett. 106, 096801 (2011).

20. Kumar, S., Huck, A. \& Andersen, U. L. Efficient coupling of a single diamond color center to propagating plasmonic gap modes. Nano Lett. 13, 1221-1225 (2013).

21. Bermúdez-Ureña, E. et al. Coupling of individual quantum emitters to channel plasmons. Nat. Commun. 6, 7883 (2015).

22. Kress, S. J. P. et al. Wedge waveguides and resonators for quantum plasmonics. Nano Lett. 15, 6267-6275 (2015).

23. Siampour, H., Kumar, S. \& Bozhevolnyi, S. I. Nanofabrication of plasmonic circuits containing single photon sources. ACS Photonics 4, 1879-1884 (2017).

24. Siampour, H., Kumar, S. \& Bozhevolnyi, S. I. Chip-integrated plasmonic cavityenhanced single nitrogen-vacancy center emission. Nanoscale 9, 17902-17908 (2017).

25. Sipahigil, A. et al. An integrated diamond nanophotonics platform for quantum-optical networks. Science 354, 847-850 (2016).

26. Kumar, S., Davydov, V. A., Agafonov, V. N. \& Bozhevolnyi, S. I. Excitation of nanowire surface plasmons by silicon vacancy centers in nanodiamonds. Opt. Mater. Express 7, 2586-2596 (2017).

27. Riedrich-Möller, J. et al. Deterministic coupling of a single silicon-vacancy color center to a photonic crystal cavity in diamond. Nano Lett. 14, 5281-5287 (2014).

28. Hepp, C. et al. Electronic structure of the silicon vacancy color center in diamond. Phys. Rev. Lett. 112, 036405 (2014).

29. Sipahigil, A. et al. Indistinguishable photons from separated silicon-vacancy centers in diamond. Phys. Rev. Lett. 113, 113602 (2014).

30. Jantzen, U. et al. Nanodiamonds carrying silicon-vacancy quantum emitters with almost lifetime-limited linewidths. New J. Phys. 18, 073036 (2016).

31. Li, K., Zhou, Y., Rasmita, A., Aharonovich, I. \& Gao, W. B. Nonblinking emitters with nearly lifetime-limited linewidths in CVD nanodiamonds. Phys. Rev. Appl. 6, 024010 (2016).
32. Iwasaki, T. et al. Germanium-vacancy singlecolor centers in diamond. Sci. Rep. 5, $12882(2015)$

33. Bhaskar, M. K. et al. Quantum nonlinear optics with a germanium-vacancy color center in a nanoscale diamond waveguide. Phys. Rev. Lett. 118, 223603 (2017).

34. Siyushev, P. et al. Optical and microwave control of germanium-vacancy center spins in diamond. Phys. Rev. B 96, 081201 (2017).

35. Palyanov, Y. N., Kupriyanov, I. N., Borzdov, Y. M. \& Surovtsev, N. V. Germanium: a new catalyst for diamond synthesis and a new optically active impurity in diamond. Sci. Rep. 5, 14789 (2015).

36. Häußler, S. et al. Photoluminescence excitation spectroscopy of $\mathrm{SiV}^{-}$and $\mathrm{GeV}^{-}$color center in diamond. New J. Phys. 19, 063036 (2017).

37. Iwasaki, T. et al. Tin-vacancy quantum emitters in diamond. Phys. Rev. Lett. 119 253601 (2017).

38. Tchernij, S. D. et al. Single-Photon-emitting optical centers in diamond fabricated upon sn implantation. ACS Photonics 4, 2580-2586 (2017).

39. Szenes, A. et al. Improved emission of SiV diamond color centers embedded into concave plasmonic core-shell nanoresonators. Sci. Rep. 7, 13845 (2017).

40. Neu, E. et al. Low-temperature investigations of single silicon vacancy colour centres in diamond. New J. Phys. 15, 043005 (2013).

41. Ditlbacher, H. et al. Silver nanowires as surface plasmon resonators. Phys. Rev. Lett. 95, 257403 (2005)

42. Nagpal, P., Lindquist, N. C., Oh, S. H. \& Norris, D. J. Ultrasmooth patterned metals for plasmonics and metamaterials. Science 325, 594-597 (2009).

43. Park, J. H. et al. Single-crystalline silver films for plasmonics. Adv. Mater. 24, 3988-3992 (2012)

44. Lu, Y. J. et al. Plasmonic nanolaser using epitaxially grown silver film. Science 337, 450-453 (2012).

45. Wu, Y. W. et al. Intrinsic optical properties and enhanced plasmonic response of epitaxial silver. Adv. Mater. 26, 6106-6110 (2014).

46. Chang, C. W. et al. $\mathrm{HNO}_{3}$-assisted polyol synthesis of ultralarge singlecrystalline ag microplates and their far propagation length of surface plasmon polariton. ACS Appl. Mater. Interfaces 6, 11791-11798 (2014).

47. Wang, C. Y. et al. Giant colloidal silver crystals for low-loss linear and nonlinear plasmonics. Nat. Commun. 6, 7734 (2015).

48. Rogers, L. J. et al. Multiple intrinsically identical single-photon emitters in the solid state. Nat. Commun. 5, 4739 (2014).

49. Hoang, T. B., Akselrod, G. M. \& Mikkelsen, M. H. Ultrafast room-temperature single photon emission from quantum dots coupled to plasmonic nanocavities. Nano Lett. 16, 270-275 (2016).

50. Rogers, L. J. et al. Electronic structure of the negatively charged silicon-vacancy center in diamond. Phys. Rev. B 89, 235101 (2014).

51. Stehlik, S. et al. Size and purity control of HPHT nanodiamonds down to $1 \mathrm{~nm}$. J. Phys. Chem. C 119, 27708-27720 (2015).

52. Stehlik, S. et al. High-yield fabrication and properties of $1.4 \mathrm{~nm}$ nanodiamonds with narrow size distribution. Sci. Rep. 6, 38419 (2016).

53. Palik, E. Handbook of Optical Constants of Solids (Academic Press, Orlando, 1985).

54. Holmgaard, T. et al. Efficient excitation of dielectric-loaded surface plasmonpolariton waveguide modes at telecommunication wavelengths. Phys. Rev. $B$ 78, 165431 (2008).

55. Holmgaard, T., Bozhevolnyi, S. I., Markey, L. \& Dereux, A. Dielectric-loaded surface plasmon-polariton waveguides at telecommunication wavelengths: excitation and characterization. Appl. Phys. Lett. 92, 011124 (2008).

56. Bozhevolnyi, S. I., Martin-Moreno, L., Garcia-Vidal, F. Quantum Plasmonics (Springer, Cham, 2017).

57. Chen, Y. T., Nielsen, T. R., Gregersen, N., Lodahl, P. \& Mørk, J. Finite-element modeling of spontaneous emission of a quantum emitter at nanoscale proximity to plasmonic waveguides. Phys. Rev. B 81, 125431 (2010).

58. Heeres, R. W., Kouwenhoven, L. P. \& Zwiller, V. Quantum interference in plasmonic circuits. Nat. Nanotechnol. 8, 719-722 (2013).

59. Fakonas, J. S., Lee, H., Kelaita, Y. A. \& Atwater, H. A. Two-plasmon quantum interference. Nat. Photonics 8, 317-320 (2014).

60. Andersen, S. K. H., Kumar, S. \& Bozhevolnyi, S. I. Ultrabright linearly polarized photon generation from a nitrogen vacancy center in a nanocube dimer antenna. Nano Lett. 17, 3889-3895 (2017). 